\title{
Adenoid Cystic Carcinoma of the Sinonasal Tract: Treatment Results
}

Chae-Seo Rhee, MD; Tae-Bin Won, MD; Chul Hee Lee, MD; Yang-Gi Min, MD; Myung-Whun Sung, MD; Kwang-Hyun Kim, MD; Woo Sub Shim, MD, Yong Min Kim, MD; Jeong-Whun Kim, MD

Objectives: Malignancies arising from the sinonasal tract, which includes the nose, paranasal sinuses, and nasopharynx, are uncommon. Although adenoid cystic carcinoma (ACC) is the second most common cancer occurring in the sinonasal tract, only few studies have been reported. This retrospective review was performed to identify the clinical features and treatment outcomes of sinonasal ACC. Methods: Thirty-five patients diagnosed and treated for ACC of the sinonasal tract were included in this study. Medical records, radiographs, and pathologic slides were retrospectively reviewed. Results: In two thirds of the patients, the maxillary sinus was the site of origin and cribriform was the most common histologic subtype (61\%). Seventy-one percent of the patients had advanced disease (T3, T4) at the time of diagnosis. Five-year overall survival rate was $86 \%$ and treatment failure occurred in 18 patients (51\%). Five-year local recurrence rate and distant metastasis rate were $30 \%$ and 25\%, respectively. Adjunctive radiotherapy appeared to reduce local recurrence. Presence of distant metastasis correlated with decreased 5-year survival $(P=$ .001). Five-year survival rate after development of distant metastasis or local recurrence were $17 \%$ and $58 \%$, respectively. Conclusions: Based on our findings, we suggest that sinonasal ACC be treated by a combined modality of radical surgery followed by postoperative radiation. The prognosis of sinonasal ACC seems to be determined by the presence of distant metastasis. Key Words: Adenoid cystic carcinoma, sinonasal tract, distant metastasis.

Laryngoscope, 116:982-986, 2006

From the Departments of Otorhinolaryngology-Head and Neck Surgery, Seoul National University Hospital (C.-S.,R., T.-B.W., Y.-G.M., M.-W.s., K.-H.K., W.S.S., Y.M.K.), Seoul, Korea, and Seoul National University Bundang Hospital (C.H.L., J.-w.K.), Seongnam, Korea.

Editor's Note: This Manuscript was accepted for publication February $17,2006$.

This study was partly supported by grant number 04-05-003 from the SNUBH Research Fund.

Drs. Rhee and Won equally contributed to the content of this article.

Send Correspondence to Dr. Jeong-Whun Kim, Associate Professor, Department of Otorhinolaryngology-Head and Neck Surgery, Seoul National University Bundang Hospital, 300 Goomi-Dong, Bundang-Gu, Seongnam-Si, Kyunggi-Do, 463-707, South Korea. E-mail: kimemail@ snubh.org

DOI: 10.1097/01.mlg.0000216900.03188.48

\section{INTRODUCTION}

The sinonasal tract is an uncommon primary site for malignant disease. The incidence of sinonasal cancer is predicted to be one in 100,000 per year. It comprises approximately $3 \%$ of all the upper aerodigestive tract malignancies and less than $1 \%$ of all the cancers. ${ }^{1,2}$ Because early manifestations mimic inflammatory diseases such as chronic rhinitis and rhinosinusitis and because the paranasal sinuses are air-filled spaces, diagnosis is often delayed and is usually found in advanced stages. The sinonasal tract adjourns functionally important structures, including the dura, brain, orbit, carotid arteries, and cranial nerves. Therefore, treatment for the advanced disease can result in serious morbidity. The most common histology of cancers arising in the nasal cavity and the paranasal sinuses is squamous cell carcinoma and is followed by malignancies of salivary gland origin. Adenoid cystic carcinoma (ACC) is the most common salivary gland tumor in the sinonasal tract ${ }^{3-5}$ and accounts for $10 \%$ to $25 \%$ of all head and neck ACC. $2,6,7$

Despite the clinical implications, owing to the low incidence of sinonasal ACC, clinical reports are lacking. The present study aimed to identify the clinicopathologic characteristics and treatment outcomes of sinonasal ACC.

\section{MATERIALS AND METHODS}

From 1979 through 2004, 35 patients treated in Seoul National University Hospital and Seoul National University Bundang Hospital under the diagnosis of ACC of the sinonasal tract were included in this study. Medical records concerning site of origin, initial and adjunctive treatment modality, and outcomes were reviewed. Also, radiographs and pathologic slides were reviewed by one head and neck radiologist and one pathologist. Eighteen males and 17 females were included in this study and their mean age was 47 years. The main clinical manifestations were cheek swelling, nasal obstruction, cheek pain, epistaxis, nasal discharge, cheek hypoesthesia, and eyeball pain. The patients were clinically staged according to the guidelines of the American Joint Committee on Cancer for each anatomic site. ${ }^{8}$ The average follow up after treatment was 64 months. To determine presence of lung metastasis, regular chest radiographs were checked every 3 months for the first 2 years and every 6 months thereafter. Disease-specific survival, local recurrence, and distant metastasis rate were analyzed using the Kaplan-Meier method and log-rank test. $P$ value less than .05 was considered statistically significant. 
TABLE I.

Clinicopathologic Characteristics of the Patients with Adenoid Cystic Carcinoma of the Sinonasal Tract Included in This Study.

\begin{tabular}{lr}
\hline Characteristics & No. of Patients \\
\hline Primary tumor site & 8 \\
Nasal cavity & 23 \\
Maxillary sinus & 1 \\
Ethmoid sinus & 3 \\
Nasopharynx & \\
Primary tumor status & 1 \\
$\mathrm{~T}_{1}$ & 9 \\
$\mathrm{~T}_{2}$ & 8 \\
$\mathrm{~T}_{3}$ & 17 \\
$\mathrm{~T}_{4}$ & \\
Nodal status & 33 \\
$\mathrm{~N}_{0}$ & 2 \\
$\mathrm{~N}_{1-3}$ & \\
Distant metastasis & 34 \\
$\mathrm{M}_{0}$ & 1 \\
$\mathrm{M}_{1}$ & \\
\hline \hline
\end{tabular}

\section{RESULTS}

\section{Clinicopathologic Characteristics of the Tumor}

The primary tumor site were the nasal cavity $(n=8)$, the ethmoid sinus $(n=1)$, the maxillary sinus $(n=23)$, and the nasopharynx $(n=3)$. Half $(n=17)$ of the patients were diagnosed as stage T4 tumor. Most of the patients did not have regional lymph node metastasis or distant metastasis at the time of diagnosis (Table I). Pathologic slides of 23 patients were reevaluated and were classified into tubular, cribriform, or solid subtypes. Cribriform subtype $(61 \%)$ was prevalent form, followed by tubular $(22 \%)$ and solid (17\%) subtypes. Perineural invasion was more frequently observed in solid subtype $(75 \%)$ compared with tubular (20\%) or cribriform (50\%) subtypes.

\section{Treatment Modalities According to the Primary Site}

Treatment modality varied according to primary tumor site (Table II). For ACC of the nasal cavity, surgical resection of the primary tumor was performed as the first-line treatment, except for stage T4 patients. Surgery consisted of medial maxillectomy or subtotal maxillectomy. Five of eight patients with ACC of the nasal cavity underwent postoperative radiation therapy. The patient with ACC of the ethmoid sinus showed skull base invasion, and craniofacial resection was performed to remove the tumor en bloc. For ACC of the nasopharynx, surgery was not performed and all patients were treated by radiation therapy alone. Eighteen of 23 patients with ACC of the maxillary sinus underwent surgical resection of the primary tumor followed by postoperative radiation. Total maxillectomy and orbital exenteration was performed in two patients in which the tumor involved periorbital tissue and craniofacial resection was performed in two patients in whom the tumor invaded the skull base.

\section{Treatment Results}

Eighteen of 35 patients (51\%) had treatment failures, and these consisted of local recurrence $(\mathrm{n}=9[26 \%])$, distant metastasis $(\mathrm{n}=4[11 \%])$, or both $(\mathrm{n}=5[14 \%])$. There was no regional lymph node metastasis after treatment (Table II). The time of local recurrence after treatment ranged from $51 \pm 64$ months. Distant metastasis developed in nine patients; six patients developed pulmonary metastasis, one bone metastasis, one liver metastasis, and one both lung and bone metastasis. Distant metastasis occurred $37 \pm 33$ months after treatment.

Five- and 10-year treatment failure rate was $42 \%$ and $73 \%$. Five- and 10 -year local recurrence rate was $30 \%$ and $57 \%$, and distant metastasis rate was $25 \%$ and $43 \%$, respectively (Fig. 1). Local recurrence rate was affected by primary tumor stage. Advanced-stage (T3 and T4) tumors were associated with higher local treatment failure compared with early-stage (T1 and T2) tumors $(P=.031)$ (Fig. 2 ). The 5 -year local recurrence rate was $42 \%$ for advancedstage tumors, whereas no recurrence developed in the early-stage tumors. In contrast, distant metastasis rate was not significantly higher in advanced-stage tumors compared with early-stage tumors $(P=.21)$.

Perineural invasion was found in 14 of 23 patients (60\%) whose pathological slides were reevaluated. However, the presence of perineural invasion did not significantly influence local recurrence rate $(P=.90)$ or distant metastasis rate $(P=.48)$.

Disease-specific survival rate and local recurrence rate appeared to be lower in patients treated with radical surgery combined with postoperative radiotherapy than in patients treated with other therapeutic modalities $(P=$ .17 and .09 , respectively). However, distant metastasis was not related to treatment modality $(P=.75)$. Overall disease-specific survival rate at 5 and 10 years was $86 \%$ and $53 \%$, respectively. Distant metastasis seemed to be an important factor in determining survival. The 5- and 10year survival rate in patients without distant metastasis were $100 \%$ and $69 \%$ respectively, whereas in patients with distant metastasis, it was only $58 \%$ and $29 \%(P=.001$ and $P=.002$ ) (Fig. 3). The mean survival period after development of distant metastasis was 25 months and the 5 -year survival rate after occurrence of distant metastasis was $17 \%$ (Fig. 4). On the other hand, local recurrence was not a determining factor for survival $(P=.89)$. The mean survival period after local recurrence was 41 months and the 5 -year survival rate after tumor recurrence was $58 \%$.

\section{DISCUSSION}

Various malignancies of salivary gland origin, including adenocarcinoma, ACC, and mucoepidermoid carcinoma, develop in the sinonasal tract. ${ }^{9}$ Among them, ACC is a malignant tumor that shows peculiar biologic characteristics. It tends to recur locally long after remission by initial treatment. Three of the patients included in our study had local recurrence 8 years after complete remission. Frequent and delayed distant metastasis is also one of the characteristics. Based on the limited data, the present study suggests that distant metastasis rate of early-stage tumors is comparable to that of advancedstage tumors. However, these biologic features are yet 
TABLE II.

Treatment Modalities of the Primary Tumor and Results.

\begin{tabular}{|c|c|c|c|c|c|}
\hline Treatment Modalities & \multicolumn{5}{|c|}{ No. of Patients } \\
\hline \multicolumn{6}{|l|}{ Nasal cavity } \\
\hline \multicolumn{6}{|l|}{$\mathrm{T}_{1}$} \\
\hline Surgery only & 1 & 1 & & & \\
\hline \multicolumn{6}{|l|}{$\mathrm{T}_{2}$} \\
\hline \multicolumn{6}{|l|}{$\mathrm{T}_{3}$} \\
\hline Surgery only & 1 & & 1 & & \\
\hline Surgery + postoperative radiation & 2 & & 2 & & \\
\hline \multicolumn{6}{|l|}{$\mathrm{T}_{4}$} \\
\hline Radiation only & 1 & & 1 & & \\
\hline \multicolumn{6}{|l|}{ Nasopharynx } \\
\hline \multicolumn{6}{|l|}{$\mathrm{T}_{3}$} \\
\hline Radiation only & 1 & & & & 1 \\
\hline \multicolumn{6}{|l|}{$\mathrm{T}_{4}$} \\
\hline Radiation only & 2 & & 1 & 1 & \\
\hline \multicolumn{6}{|l|}{ Maxillary sinus } \\
\hline \multicolumn{6}{|l|}{$\mathrm{T}_{2}$} \\
\hline Surgery + postoperative radiation & 6 & 5 & 1 & & \\
\hline \multicolumn{6}{|l|}{$\mathrm{T}_{3}$} \\
\hline Surgery + postoperative radiation & 4 & 4 & & & \\
\hline
\end{tabular}

unexplainable. The unexpected biologic behavior of this tumor presents a great challenge, often resulting in treatment failures.

In accordance with other studies, two thirds of sinonasal ACCs in our study occurred in the maxillary sinus and the most common pathologic subtype was cribriform. The reason for the prevalence of the maxillary sinus as the primary tumor site is unknown. Pathologically, the solid subtype has more tumor cells than tubular or cribriform subtypes and is usually known to show the worst prognosis among the three subtypes. ${ }^{2,10-12}$ However, whether the pathologic subtype can serve as an independent prognosisdetermining factor in sinonasal ACC is unknown. In the present study, as a result of the small number of patients in each subtype, we could not determine whether the solid subtype serves as a poor prognosticator, and future studies to clarify the influence of pathologic subtype are needed.
Perineural invasion in ACC has been considered as one of the determining factors for locoregional recurrence or distant metastasis. ${ }^{13,14}$ However, our study did not show significant correlation between perineural invasion and local recurrence and distant metastasis.

Surgical resection with postoperative adjuvant radiation therapy was the main therapeutic modality for ACC of the nasal cavity and maxillary sinus. Although radiation therapy is known to decrease local recurrence, its contribution to survival is unclear, maybe because of the high rate of distant metastasis. ${ }^{2,5}$ In the present study, local recurrence rate was high in advanced-stage (T3 and T4) tumors despite postoperative radiation therapy in most of the patients. Therefore, complete resection of the primary tumor with sufficient tumor-free margin is recommended. For instance, en bloc craniofacial resection, including sinonasal and intracranial lesions for T4 lesions with intracranial invasion, ${ }^{15}$ is advised over conservative 


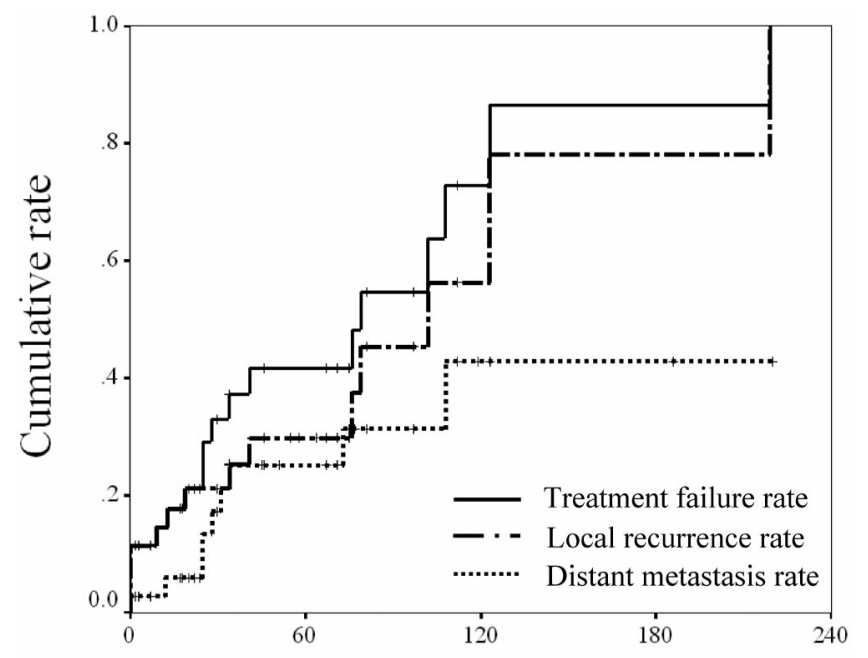

Duration (Month)

Fig. 1. Treatment failures. Treatment failure, including local recurrence and distant spread, occurred in $51 \%$ of the patients. Five-year local recurrence and distant metastasis rates were $30 \%$ and $25 \%$, respectively.

resection. Radiotherapy alone does not appear to cure the disease but probably plays a role in palliation. However, chemotherapy did not result in palliation. Considering the fact that most of the patients with sinonasal ACC were diagnosed with advanced stages, further clinical trials using multimodality therapeutic approaches aiming to reduce local recurrence are warranted. ${ }^{16}$

The 5-year survival rate was $86 \%$, although the initial treatment failed in half of the patients included in this study. This was attributed by delayed local recurrence and distant metastasis. Other studies report treatment failure rates ranging from $60 \%$ to $70 \%$ and 5-year survival rate

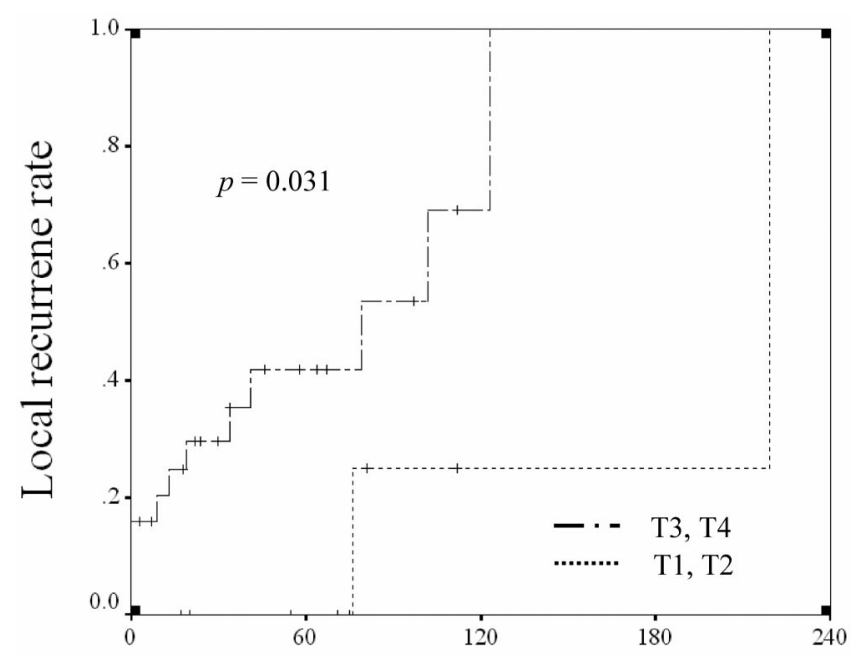

\section{Duration (month)}

Fig. 2. Local recurrence rate. Advanced-staged tumors $\left(T_{3}, T_{4}\right)$ locally recurred more frequently than early-staged tumors $\left(\mathrm{T}_{1}, \mathrm{~T}_{2}\right)(P$ $=.031$ ).

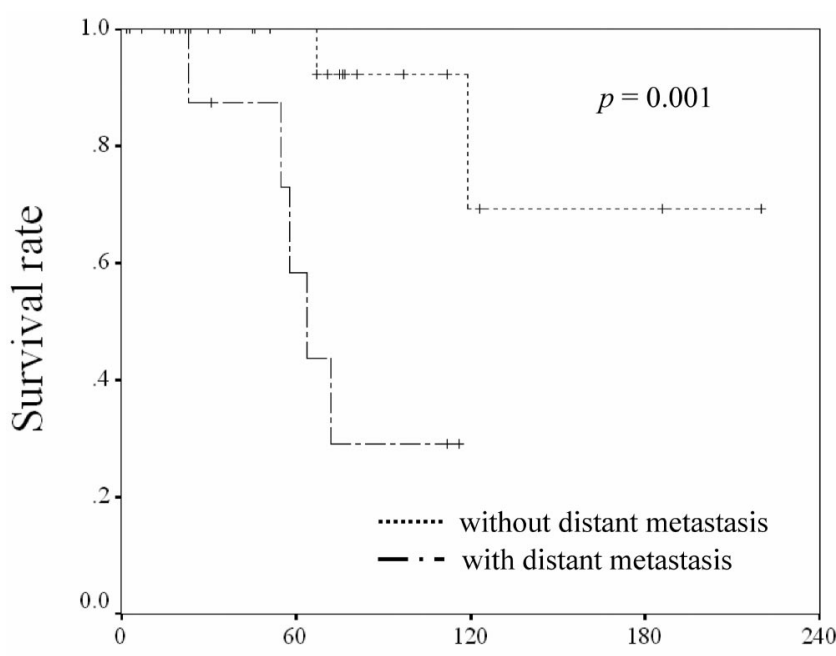

\section{Duration (month)}

Fig. 3. Disease-specific survival rate. Distant metastasis of sinonasal adenoid cystic carcinoma predicted a poorer prognosis $(P=$ $.001)$.

around 50\%.5,17 The better results shown in the present study might be explained by the lower rate of distant metastasis compared with other studies ${ }^{17}$ and by the use of radical resection as the surgical modality. Although the overall distant metastasis rate of sinonasal ACC was lower compared with other studies, the determining factor for survival was also the presence of distant metastasis and not local recurrence.

ACC is different from squamous cell carcinomas of the head and neck in that cervical lymph node metastases are uncommon. However, the presence of lymph node metastasis is regarded as a poor prognostic factor to distant

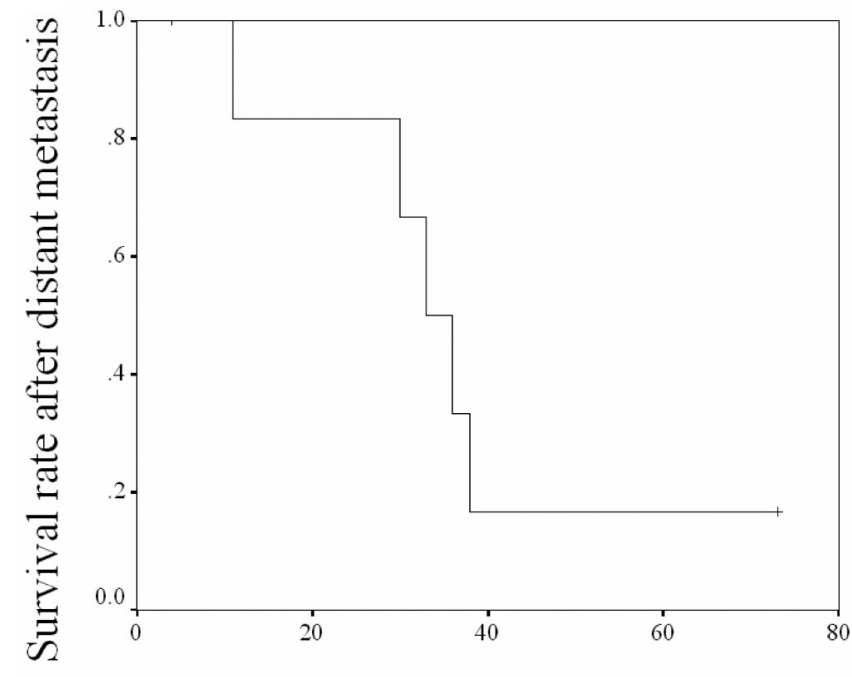

Duration (month)

Fig. 4. Disease-specific survival rate after occurrence of distant metastasis. After distant metastasis developed, 5-year survival rate was $17 \%$ and median survival period was 25 months. 
metastasis and survival. ${ }^{17}$ Other studies have reported occurrence of regional lymph node metastasis in $10 \%$ to $15 \%$ of head and neck ACC. ${ }^{2,6,17}$ In the present study, lymphatic spread was found in only two patients $(6 \%)$ at the time of diagnosis and regional nodal recurrence did not occur. The low rate of nodal metastasis for sinonasal ACC compared with other ACCs of the head and neck might be accounted for by the sparse lymphatic distribution in the sinonasal tract and surrounding structures.

In summary, based on the limited data, the present study suggests that whenever sinonasal ACC is resectable, radical surgical resection and adjuvant postoperative radiotherapy is recommended to reduce local recurrence rate. Although distant metastasis is uncommon in sinonasal ACC compared with ACC of other head and neck areas, it seems to determine survival. In the future, a multimodality treatment approach, including adjuvant chemotherapy, surgery, and radiation therapy, is warranted to further prevent local recurrence and distant metastasis.

\section{BIBLIOGRAPHY}

1. Witt RL. Adenoid cystic carcinoma of the minor salivary glands. Ear Nose Throat J 1991;70:218-222.

2. Sung MW, Kim KH, Kim JW, et al Clinicopathologic predictors and impact of distant metastasis from adenoid cystic carcinoma of the head and neck. Arch Otolaryngol Head Neck Surg 2003;129:1193-1197.

3. Batsakis JG, Luna MA, El-Naggar A. Histopathologic grading of salivary gland neoplasms: III-adenoid cystic carcinoma. Ann Otol Rhinol Laryngol 1990;99:1007-1009.

4. Goepfert H, Luna MA, Lingberg RD, White AK. Malignant salivary gland tumors of the paranasal sinuses and nasal cavity. Arch Otolaryngol 1983;109:662-668.
5. Bhattacharyya N. Factors affecting survival in maxillary sinus cancer. J Oral Maxillofac Surg 2003;61:1016-1021.

6. Jones AS, Hamilton JW, Rowley H, et al. Adenoid cystic carcinoma of the head and neck. Clin Otolaryngol 1997;22: $434-443$.

7. Spiro RH, Huvos AG, Hicks WF, et al. Stage means more than grade in adenoid cystic carcinoma. Am J Surg 1992; 164:623-628.

8. American Joint Committee on Cancer. Manual for Staging of Cancer, 6th ed. Philadelphia: JB Lippincott; 2002.

9. Harbo G, Grau C, Bundgaard T, et al Cancer of the nasal cavity and paranasal sinuses: clinico-pathological study of 277 patients. Acta Oncol 1997;36:45-50.

10. Perzin KH, Gullane P, Clairmont AC. Adenoid cystic carcinoma in the salivary glands: a correlation of histologic features and clinical course. Cancer 1978;42:265.

11. Cleveland D, Abrams AM, Melrose RJ, Handlers JP. Solid adenoid cystic carcinoma of the maxilla. Oral Surg Oral Med Oral Pathol 1990;69:470-478.

12. Szanto PA, Luna MA, Tortoledo ME. Histologic grading of adenoid cystic carcinoma of the salivary glands. Cancer 1984;54:1062-1069.

13. Van der Wal JE, Snow GB, Van der Wal I. Intraoral adenoid cystic carcinoma, the presence of perineural spread in relation to site, size, local extension and metastatic spread. Cancer 1990;66:2031-2033.

14. Teymoortash A, Pientka A, Schrader C, Tiemann M, Werner JA. Expression of galectin-3 in adenoid cystic carcinoma of the head and neck and its relationship with distant metastasis. J Cancer Res Clin Oncol 2005;24:1-6.

15. Pitman KT, Prokopakis EP, Aydogan B, et al The role of skull base surgery for the treatment of adenoid cystic carcinoma of the sinonasal tract. Head Neck 1999;21:402-427.

16. Day TA, Beas RA, Schlosser RJ, et al Management of paranasal sinus malignancy. Curr Treat Options Oncol 2005;6: $3-18$.

17. Spiro RH. Distant metastasis in adenoid cystic carcinoma of salivary origin. Am J Surg 1997;174:495-498. 